\title{
Ortomonstro: um audiogame móvel customizável para práticas ortográficas de português por meio do Braille
}

\author{
Maria C. C. Araújo ${ }^{1,3}$, Bruno Roberto da Silva ${ }^{2}$, Jonathan Garcia de Queiroz ${ }^{1,3}$ e \\ Windson Viana ${ }^{1,3}$ \\ ${ }^{1}$ Grupo de Redes de Computadores, Engenheria de Software e Sistemas - Fortaleza - CE \\ ${ }^{2}$ Instituto Federal de Educação, Ciência e Tecnologia do Ceará - Fortaleza - CE \\ ${ }^{3}$ Universidade Federal do Ceará - Fortaleza - CE \\ maria.araujo@great.ufc.br, bruno.robertodifce.edu.br \\ jonathan.garciaegreat.ufc.br, windsonegreat.ufc.br
}

\begin{abstract}
Serious Games and e-learning applications for mobile platforms have great potential for improving teaching and learning processes, however, they contain significant challenges for the implementation of accessibility mechanisms. In this context, we developed an accessible mobile audiogame for the visually impaired people, called Ortomonstro. The games is focused on teaching ortografy by using the Braille system. The audiogame was initially designed to assist in the use and practice of Braille with ortografics exercises of Portuguese. A Web interface provides customization of questions and audiogame contents. An usability and acessibility evaluation was implemented with five users. Initial results indicate good acceptance by users and teachers.
\end{abstract}

Resumo. Jogos sérios e aplicações educativas para plataformas móveis apresentam grande potencial de melhoria dos processos de ensino-aprendizagem, porém, ainda é um desafio tornar acessíveis as interfaces dessas aplicações. Nesse contexto, foi desenvolvido um audiogame móvel acessivel para pessoas com deficiência visual, chamado Ortomonstro, voltado para práticas pedagógicas de ortografia usando o sistema Braille. O audiogame é composto de um aplicação móvel Android e de uma interface Web de customização. A interface Web proporciona a geração de customização dos conteúdos do audiogame. Uma avaliação de usabilidade e acessibilidade foi realizada e, à princípio, a aceitação de usuários e professores com esse tipo de solução é positiva.

\section{Introdução}

Além de entretenimento, os jogos digitais são cada vez mais utilizados para outros fins, como a educação, treinamento e melhoria da saúde. Jogos com temática educacional podem ser classificados como jogos sérios, isto é, jogos cujo game design já prevê uma intenção de ser mais do que puro entretenimento (e.g., explicar um período Histórico, exercitar um conceito matemático) [U. RITTERFELD and Wong. 2009]. Esse sucesso dos jogos digitais e seus desdobramentos aumentaram sua importância e a necessidade da sua universalização. No Brasil, de acordo com informações do Instituto Brasileiro de Geografia e Estatística - IBGE, o último Censo, que data de 2010, aponta que o Brasil tem 45,6 milhões de pessoas com deficiência. A deficiência mais frequente entre a população 
V Congresso Brasileiro de Informática na Educação (CBIE 2016)

Anais do XXVII Simpósio Brasileiro de Informática na Educação (SBIE 2016)

brasileira é a visual. Cerca de 35 milhões de pessoas $(18,8 \%)$ declararam ter dificuldade de enxergar, mesmo com óculos ou lentes de contato [IBGE 2010].

Apesar da importância do desenvolvimento de jogos acessíveis, um número significativo de pessoas ainda encontra barreiras ao acessar jogos devido a algum tipo ou contexto de deficiência. Os problemas relacionados à acessibilidade podem incluir, entre outros, ausências de feedbacks; tratamento deficiente de dados; e interações inadequadas com dispositivos de entrada e saída convencionais [Maria C. C. Araújo 2015]. Uma abordagem que tenta dirimir esses problemas para pessoas com deficiência visual são os denominados audiogames, isto é, jogos acessíveis focados em soluções de áudio.

Os audiogames que se voltam para temáticas com intuito educativo podem ser descritos como audiogames sérios. E, neste caso, os audiogames sérios envolvem objetivamente duas problemáticas principais: o desenvolvimento e construção do aprendizado para pessoas cegas por meio do jogo e as implementações voltadas para soluções de inclusão e acessibilidade nesses jogos. Atualmente, ainda são tímidos os esforços existentes para a produção deste tipo de jogo [Milne et al. 2014], [Silva et al. 2014], tanto nas plataformas Web de educação a distância, quanto nas que fazem uso da Web em combinação com dispositivos móveis.

Nesse sentido, o objetivo principal deste trabalho foi desenvolver e avaliar um audiogame sério e acessível: o Ortomonstro. O jogo envolve a prática do Braille para o exercício e aprendizado de ortografia. Essas práticas podem ser customizadas por professores por meio da adaptação de itens e configurações disponíveis em uma interface Web. Esta contém ferramentas e opções para a customização do audiogame no dispositivo móvel dos alunos.

O restante deste artigo está organizado da seguinte forma: a Seção 2 apresenta a Fundamentação Teórica em que são descritos conceitos sobre audiogames e acessibilidade. Na Seção 3, os trabalhos relacionados são relatados. Na Seção 4, é descrito o funcionamento e a implementação do jogo. Na Seção 5, está relatada a avaliação inicial de usabilidade e acessibilidade. E, por fim, a Seção 6 traz a conclusão e trabalhos futuros.

\section{Fundamentação Teórica}

\subsection{Audiogames e Acessibilidade}

A característica principal dos audiogames é o tratamento das rotinas e narrativas do jogo por meio de recursos sonoros e informações textuais que podem ser lidas por leitores de tela. Esse tipo de game quer que o jogador tenha a capacidade de diferenciar em tempo hábil vários padrões de áudio distintos. Para tornar mais completo esse processo, têm-se três principais modalidades de acesso aos retornos gerados pelos audiogames, a saber: retorno principal de áudio, retorno tátil e retorno háptico (retornos com vibração e/ou som). Audiogames podem ter design universal, e assim, inserir pessoas sem deficiência visual no jogo. Nesse caso, a interação ideal deve conter também interfaces visuais.

Os recursos de acessibilidade encontrados na maioria dos audiogames limitam-se, em geral, à alteração da resolução, ajuste dos volumes, ativação de legendas e remapeamento dos controles [Thorpe et al. 2011]. Outros exemplos de acessibilidade seriam as técnicas denominadas de AudioQuake e Serialization [Maria C. C. Araújo 2015]. Ainda que esses mecanismos representem um espaço importante na promoção de um design 
V Congresso Brasileiro de Informática na Educação (CBIE 2016)

Anais do XXVII Simpósio Brasileiro de Informática na Educação (SBIE 2016)

universal em audiogames acessíveis, as necessidades impostas por limitações mais severas ainda são desatendidas: incompatibilidade com Tecnologias Assistivas (como acionadores ou leitores de tela), falta de feedback multimodal (como vibração de controles) e ausência de legendas ocultas (closed caption). Os autores [Maria C. C. Araújo 2015] e [Cheiran and Pimenta 2011] discorrem sobre essas técnicas e indicam recomendações mais comuns para promover acessibilidade neste tipos jogos. As principais técnicas foram implementadas no jogo Ortomonstro para garantir maior chance de prover boa acessibilidade e usabilidade no mesmo.

\subsection{Objetos de Aprendizagem e customização}

De acordo com o Learning Technology Standards Committee (LTSC), Objetos de Aprendizagem (OA's) são descritos como "qualquer entidade, digital ou não digital, que possa ser usada, reutilizada ou referenciada durante o aprendizado suportado por tecnologias". Assim, um OA pode ser definido como uma unidade de instrução/ensino reutilizável [Tarouco et al. 2004].

O ambiente de customização Web do audiogame móvel construído neste trabalho está relacionado mais objetivamente a um tipo específico de objeto de aprendizagem, denominado de Objeto de Aprendizagem Customizável (OAC). O OAC difere do OA porque este permite que modificações sejam realizadas diretamente em uma interface do recurso sem necessitar, por parte do professor [de Souza et al. 2012], nenhum conhecimento de programação. Assim, um OAC é um OA com permissões de customização atreladas aos elementos que compõem sua interface. Essas modificações são norteadas por uma estratégia de adaptação adotada ainda na fase de construção do recurso de customização que priorizam a autonomia do professor, que facilmente pode ser ampliada com o uso dos OAC's, tendo em vista que ao fazer uso de recursos customizáveis, o próprio professor delimita a sequência a ser explorada com o jogo em suas aulas.

\section{Trabalhos Relacionados}

Existem muitos esforços atualmente para produzir recursos educacionais acessíveis. Alguns trabalhos tratam especificamente de adaptar adequadamente aplicativos móveis e softwares leitores de tela, que reproduzem texto escrito no formato de áudio, e que são exemplos de excelente suporte para o aprendizado de pessoas cegas. Trabalhos envolvendo adaptação de leitura para reprodução de símbolos matemáticos para o Braille [Meira et al. 2008], e outros tratando da adaptação de jogos, como o jogo da forca para praticar ortografia com o Braille são alguns exemplos [Silva et al. 2014].

Outro exemplo de pesquisa que se aproxima do contexto dessa proposta é o BraillePlay [Milne et al. 2014], o qual consiste em um conjunto de jogos acessíveis para smartphones que ensinam as codificações dos caracteres em Braille, a fim de promover a alfabetização no sistema Braille. A suíte é composta por quatro jogos: VBReader, VBWriter, VBHangman, e VBGhost. Os primeiros dois jogos são jogos com cartas cinzas em que se exercitam as habilidades dos usuários para reconhecer e escrever caracteres Braille. No VBHangman, o usuário joga contra o aplicativo e tenta deduzir qual é a palavra, dado o seu comprimento e utilizando um número limitado de palpites. E no VBGhost, os usuários se revezam acrescentando letras para um fragmento de palavra. Um dos problemas do BraillePlay e de outros similares é uma ausência de meios para customização das 
V Congresso Brasileiro de Informática na Educação (CBIE 2016)

Anais do XXVII Simpósio Brasileiro de Informática na Educação (SBIE 2016)

atividades propostas pelos jogos. Considerando isto, um ambiente denominado GBraille, proposto em [Araújo et al. 2016], traz a proposta de prover customização de atividades e ser facilmente adaptado para suportar outros idiomas (como Espanhol e Inglês). O principal objetivo do ambiente GBraille é fornecer um conjunto de jogos para aprendizagem móvel também com foco no uso e prática do Braille para exercitar a ortografia.

Dois jogos do ambiente GBraille estão fortemente relacionados ao Ortomonstro: GBraille Hangman e GBraille Asteroids. Os dois jogos também possuem a temática de exercício da ortografia por meio do teclado GBraille Keyboard. Nesse sentido, o Ortomonstro é uma evolução dos trabalhos apresentados no ambiente GBraille ampliando a gama de audiogames disponíveis no mesmo e provendo um ciclo completo de customização por parte dos professores.

\section{Ortomonstro}

\subsection{Princípios de Design}

O design do jogo foi definido após entrevistas com professores de educação especial que possuíam experiência com ensino do Braille. Uma análise de jogos similares de formato acessível também foi efetuada. O Ortomonstro foi desenhado de forma a se integrar às práticas pretendidas pelos professores em torno de suas próprias perguntas e respostas. O método para a construção do jogo foi conduzido com base no processo Human-centred design for interactive systems [DIS 2009], o qual é conduzido e refinado pela avaliação centrada nos usuários e aborda toda a experiência descrito por estes. $\mathrm{O}$ jogo possui dois níveis de dificuldade e auxilia alunos na aquisição de conhecimentos nos tópicos de ortografia contemplados pelo jogo (e.g. Sinônimos, Acentuação, Plurais e Ditados). Uma amostra de vídeo do funcionamento do jogo está disponível em http://tinyurl.com/js3z6k5

A Figura 1 apresenta o esquema de funcionamento do jogo, que é composto de um aplicativo móvel e uma interface Web de customização que provê configurações enviadas ao dispositivo móvel. Esta arquitetura tem a finalidade de transformar o jogo em um OAC. Nesse sentido, o sistema apresenta algumas características referentes a princípios de design de OAC's, tais como: construção focada em customização de itens (e.g., modificar perguntas e respostas, personalizar conteúdo multimídia, habilitar/desabilitar níveis do jogo), bem como uma construção baseada em práticas de reusabilidade de software. A Figura 2 reflete uma simplificação do diagrama de classes do jogo. Podem ser vistos os elementos que são específicos e fixos no jogo, classes que definem elementos customizáveis e classes que definem elementos reutilizáveis, como o GBraille Keyboard e os Menus. O GBraille Keyboard é um aplicativo multimodal que permite a entrada de dados em Braille em dispositivos touchscreen [Silva et al. 2014]. Nele, um símbolo Braille pode ser inserido em três passos e com poucos toques, deixando mais rápido o processo de escrita. A versão mais recente do teclado é apresentada na terceira tela da Figura 3.

\subsection{Gameplay}

O Ortomonstro é um audiogame educativo que utiliza perguntas, respostas e desafios bônus. O objetivo do jogador é não colecionar monstros. Em cada jogada é exibida uma pergunta, e o jogador precisa repassar uma palavra completa ou parte da palavra como resposta, a depender do nível do jogo, inserindo todas as letras, uma por vez. As perguntas 


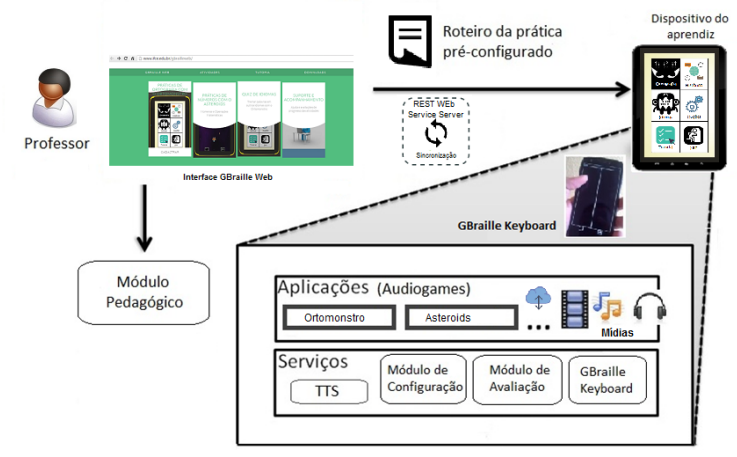

Figura 1. Esquema de funcionamento do jogo Ortomonstro

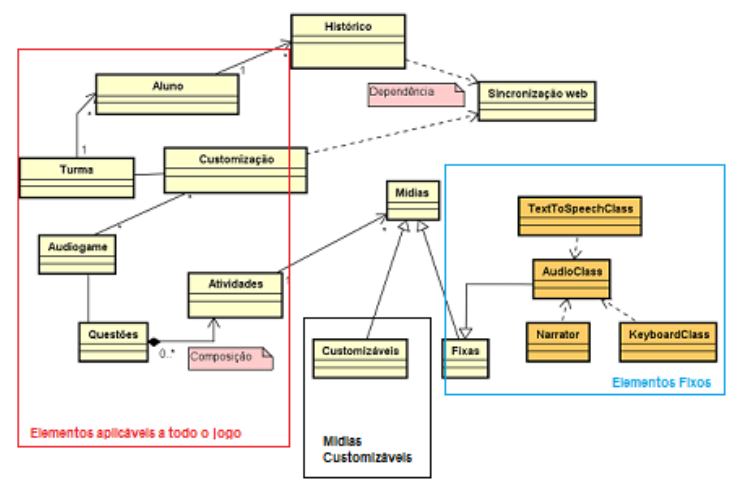

Figura 2. Modelo de classes do Ortomonstro

não obedecem a uma sequência pré-definida de exibição, aumentando o desafio. Durante cada pergunta, o jogador terá duas chances de acertar a resposta. A cada dois erros o jogador acumula um monstro ortográfico. Ao acumular três monstros ortográficos, ou seja, a cada seis erros, o jogador é preso na caverna do léxico ortográfico, precisando de chances de obter as moedas "escudo". através de perguntas do tipo "premium". As moedas "escudo"se forem acumuladas em número de quatro podem ser convertidas em pontos extras nas atividades desenvolvidas pelos professores. Se o jogador estiver próximo de atingir a pontuação negativa que o leva à caverna do léxico, perguntas "premium" ou com opções de "ajuda"podem ser sorteadas para dispor de dicas sobre os caracteres solicitados e de uma explicação sobre o tipo de atividade ortográfica que está em prática. Assim, os jogadores podem refletir sobre o resultado de suas ações e avaliar melhor possíveis correções a realizar.

O jogo é dividido em dois módulos: Módulo de Ortografia e Modulo de Idiomas, que são escolhidos de acordo com a prática desejada pelo professor na interface Web. No módulo ortográfico, o jogador tem que escrever corretamente em Braille a palavra ou trecho de letras sorteada pelo jogo. Nesse módulo é possível acessar perguntas em níveis fácil ou difícil para sinônimos, antônimos, plural, acentuação e ditado de palavras. O módulo de idiomas segue as mesmas práticas, mas basicamente com o objetivo de treinar outra língua escolhida pelo professor que não seja o português (ver Figura 3). Além desses dois módulos, descritos na tela inicial, existem também as opções de menu para acessar um tutorial básico de utilização do jogo, um menu para o histórico de pontuação e atividades do usuário, um menu de opções, onde é possível acessar configurações da 
V Congresso Brasileiro de Informática na Educação (CBIE 2016)

Anais do XXVII Simpósio Brasileiro de Informática na Educação (SBIE 2016)

conta do usuário e atualizar o banco de perguntas disponível para o jogo e há também na tela inicial uma opção de sair do jogo.
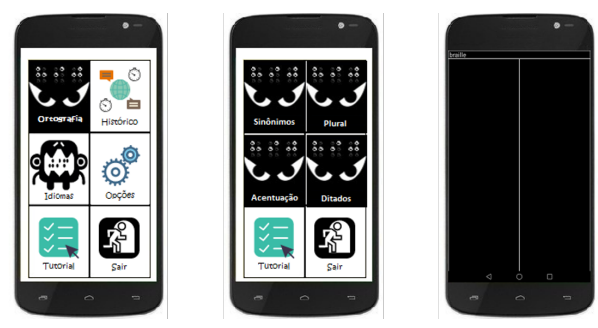

Figura 3. Telas do jogo Ortomonstro.

\subsection{Interface de Customização}

A Figura 4 apresenta telas da interface Web de customização. A primeira tela é apresentada ao professor após logar-se no sistema. Após o login, o professor visualiza duas seções iniciais. A primeira seção disponibiliza ao professor um formulário para inserção de perguntas no jogo no Módulo Ortográfico. O formulário apresenta itens customizáveis para as atividades, como por exemplo, a que tema as perguntas se referem, a que fase ou nível do jogo pertence e etc, conforme pode ser visualizado na segunda e terceira telas da Figura 4. Nas opções de cadastro das perguntas outros tipos de elementos ou mídias podem ser inseridos para situar e tornar lúdica a experiência do jogador como o trecho de "ajuda", áudios, vídeos ou imagens com descrição.

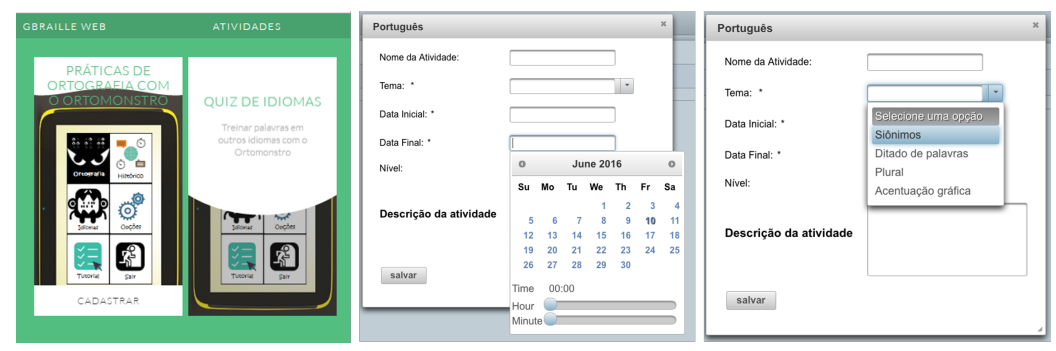

Figura 4. Páginas da Interface Web.

\section{Avaliação Inicial}

O objetivo deste trecho é relatar o processo avaliativo e os resultados preliminarmente obtidos acerca da acessibilidade e usabilidade do audiogame ortomonstro junto aos alunos e aos professores da educação especial. O jogo foi avaliado por dois professores e três alunos, visando duas questões de pesquisa principais:

Questão 1 - Como criar audiogames envolvendo Braille quem possam ser bem aceitos por usuários com deficiência visual?

Questão 2 - Como permitir que professores envolvidos com educação especial pudessem adaptar práticas ortográficas voltadas para esses esses tipos de jogos? 
V Congresso Brasileiro de Informática na Educação (CBIE 2016)

Anais do XXVII Simpósio Brasileiro de Informática na Educação (SBIE 2016)

\subsection{Usuários}

As Tabelas 1, e 2 apresentam os perfis dos usuários e professores que avaliaram as aplicações. A avaliação do jogo foi realizada em duas fases e aplicada nos dois grupos com perfis distintos. O primeiro grupo foi composto por dois usuários cegos e um usuário com baixa visão, sendo estes caracterizados como público final do jogo tanto por utilizarem com frequência smartphones e tablets, quanto por terem sido alfabetizados em Braille. O segundo grupo foi composto por duas professoras, que possuem experiência com educação especial no ensino e alfabetização com o sistema Braille em seu formato tradicional. Elas avaliaram tanto o jogo, quanto a interface web de customização.

\begin{tabular}{|l|l|l|l|}
\hline Gênero & masculino & masculino & feminino \\
\hline Idade & 37 & 29 & 26 \\
\hline Perfil & cego & cego & baixa visão \\
\hline Domínio do Braille & avançado & avançado & intermediário \\
\hline Uso de dispositivos móveis & diariamente & diariamente & diariamente \\
\hline
\end{tabular}

Tabela 1. Perfil dos usuários

\begin{tabular}{|l|l|l|}
\hline Gênero & feminino & feminino \\
\hline Idade & 41 & 39 \\
\hline Experiência em anos & 12 & 9 \\
\hline Domínio do Braille & avançado & avançado \\
\hline Uso de dispositivos móveis & diariamente & diariamente \\
\hline
\end{tabular}

Tabela 2. Perfil das profissionais de educação inclusiva

\subsection{Materiais e Métodos}

Para a avaliação inicial, o jogo foi apresentado aos usuários em um smartphone Motorola Moto G ( $2^{\text {a }}$ Geração), executando a versão 5.0.2 do sistema Android e utilizando o sintetizador de voz Loquendo TTS. Para interface Web, o acesso de deu através do navegador Google Chrome, versão 51.0.27. Um questionário foi elaborado e aplicado junto aos usuários com deficiência visual após a experiência com o jogo. O questionário foi criado com base nos trabalhos de SAVI et al. (2010). Link para o questionário aplicado aos usuários: http://tinyurl.com/hmc3gja. Outro questionário foi desenvolvido e aplicado para os profissionais especializados no ensino/aprendizagem do Braille, contendo oito perguntas relacionadas à aceitação e adequação do jogo e da interface Web na visão dos professores. Link para o questionário aplicado aos professores: http://tinyurl.com/jdczx7u.

$\mathrm{Na}$ entrevista dos usuários com deficiência visual foi dado um tempo de quinze minutos para a interação livre com o jogo, sugerindo-se que eles explorassem primeiramente o áudio contido no menu de tutorial. Após essa exploração livre, foi dado dez minutos para que o usuário conseguisse responder ao menos uma pergunta nos níveis fácil e difícil de alguma das quatro atividades ortográficas disponibilizadas. Em seguida, foi aplicado o questionário descrito anteriormente. Na entrevista com os professores, procedimento similar foi adotado. Foi oferecido dez minutos de interação livre com a interface Web antes da aplicação do questionário. Em seguida, a mesma quantidade de tempo foi fornecida para que os professores inserissem pelo menos três questões em alguma das quatro atividades de ortografia disponíveis. Ficaram todos livres para repetir o procedimento e em seguida observar o resultado da customização das perguntas no dispositivo móvel, sendo fornecido mais quinze minutos para a interação com o jogo. 
V Congresso Brasileiro de Informática na Educação (CBIE 2016)

Anais do XXVII Simpósio Brasileiro de Informática na Educação (SBIE 2016)

\subsection{Resultados e Discussões}

$\mathrm{Na}$ avaliação inicial, a interface do jogo Ortomonstro agradou a todos os usuários, todos conseguiram realizar os passos sugeridos no tempo fornecido, tanto os jogadores com deficiência visual quanto as professoras. Apenas um das pessoas com deficiência relatou que, embora tenha ouvido as instruções básicas do tutorial, sentiu uma falta de ambientação no jogo para iniciar e entender a narrativa de acertos, a sistemática da pontuação e sobrevivência no jogo sem cair na "caverna do léxico". Os jogadores conseguiram compreender a disposição dos elementos na tela, embora dois deles relataram que algumas informações importantes para a ambientação no jogo ainda não estavam descritas adequadamente nos menus internos por meio da síntese de voz, eles sugerem que a descrição de algumas opções nos menus seja mais curta. Os usuários relataram satisfação com o uso do jogo. Porém, todos foram unânimes sobre as configurações de ajuste de velocidade de reprodução das perguntas. Eles gostariam de escolher esse ajuste em um nível qualquer, mas isso está programado de acordo com a dificuldade da fase e não dos itens de perguntas isoladamente. Mesmo assim, a sugestão foi acatada e ajuste necessário para este ponto está sendo avaliado para uma próxima versão.

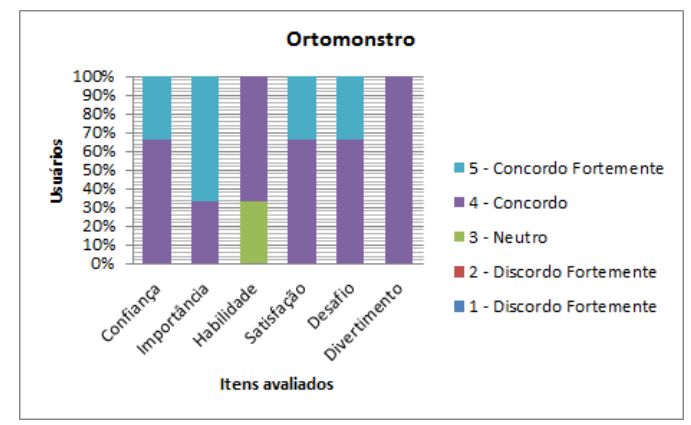

Figura 5. Gráfico de avaliação com usuários

No geral, o jogo Ortomonstro foi bem aceito, como demonstra um extrato da avaliação de acordo com a Figura 5. Entretanto, ele é ainda passível de melhorias em alguns quesitos de acessibilidade, como ajuste de velocidade de reprodução do leitor de tela personalizado pelo jogador, um guia mais detalhado da narrativa de ambientação e feedback mais adequado do sistema de pontuação, bônus e chances disponíveis. Também foi considerado agradável, divertido e adequado para a prática educativa de exercitar o Braille com a inserção dos caracteres no GBraille Keyboard. Todos os entrevistados consideraram o jogo construído e as atividades de customização associadas a ele muito pertinentes para a prática do sistema Braille e para a inserção de pessoas com deficiência visual na interação com dispositivos móveis. À princípio, as observações e testes com usuários no jogo apontam boa aceitação e adequação para qualquer perfil de usuário já alfabetizado em Braille.

Para as professoras de educação especial, o formato do jogo também seria adequado para crianças cegas que já praticassem o Braille após a fase de alfabetização, entre oito e onze anos de idade, e não somente para adultos que aprenderam o Braille e não desejam perder o contato com esta escrita. Perguntadas sobre a aceitação do modo como os caracteres são incluídos pelo teclado elas descreveram a interface do GBraille como uma boa escolha e adequada no sentido de que não acreditam ser necessária grande ex- 
V Congresso Brasileiro de Informática na Educação (CBIE 2016)

Anais do XXVII Simpósio Brasileiro de Informática na Educação (SBIE 2016)

periência ou prática com o Braille para construir cognitivamente a lógica proposta pelo teclado.

De acordo com as professoras, um ganho importante nesse jogo foi poder disponibilizar como elemento de avaliação das práticas o histórico de atividades dos alunos e poder delimitar períodos de duração adequados para estas atividades no momento da construção da prática. Para elas, interessa muito poder validar as avaliações com um histórico e poder modificar os formatos e assuntos no estilo de quiz, como ocorre tanto no módulo ortográfico, quanto no de idiomas. Quanto à interface Web, as professoras sugeriram vários pontos de melhoria como apresentar os históricos de acertos em formatos de gráficos, adicionar campos opcionais e de descrição no formulário e dispor de uma seção de atividades pré-fabricadas como modelos. Nesse sentido, existiram sugestões de uma espécie de banco de perguntas relacionadas a outros temas/matérias, que também impliquem universo de significado e formação de conhecimento que não seja puramente a língua portuguesa ou estrangeira.

\section{Conclusão e Trabalhos Futuros}

Este trabalho apresentou a descrição da construção do audiogame educativo Ortomonstro, voltado para o público com deficiência visual, e que, de acordo com avaliações iniciais, apresenta um bom nível de acessibilidade e usabilidade. O trabalho apresenta também a implementação de uma interface Web voltada para a customização desse audiogame seguindo princípios de OACs.

O desenvolvimento da interface Web como parte do audiogame visou a possibilidade de adaptação de conteúdo por parte dos professores envolvidos nas práticas de ortografia, considerando as particularidades inerentes ao processo de ensino e aprendizagem na educação especial de pessoas com deficiência visual. Tal modelo pretende ainda promover a dinamicidade em jogos educacionais acessíveis, bem como incorporar recursos que possibilitem a avaliação do desempenho dos aprendizes e, possibilitar a reutilização de alguns elementos implementados em novos jogos utilizando o modelo proposto. Como trabalhos futuros pretende-se estender a quantidade de usuários nas amostras de testes e implementar testes de acessibilidade também na interface Web, de modo a permitir a interação de professores também cegos na educação inclusiva.

\section{Agradecimentos}

Este trabalho é fruto de pesquisas desenvolvidas pela Universidade Federal do Ceará, em parceria com a Universidade do Chile e o Instituto Federal de Educação, Ciência e Tecnologia do Ceará - IFCE. Os resultados destas pesquisas fazem parte do projeto GBraille (MCT / CNPq 14/2013 - Universal), sob número de concessão 484.255 / 20134, e MCTI-SECIS/CNPq No 84/2013 - Tecnologia Assistiva, registrado sob o número de concessão 458825/2013-1 sob o Título: Pesquisa e desenvolvimento de soluções digitais para a educação, cultura e interação com sistemas móveis para pessoas com deficiência visual. Os dois projetos são apoiados pelo Programa STIC-AmSud-CAPES / CONICYT / MAEE, Projeto KIGB-Knowing and Interacting while Gaming for the Blind, 2014.

\section{Referências}

[Araújo et al. 2016] Araújo, M. C., Silva, A. R., Darin, T. G., de Castro, E. L., Andrade, R., de Lima, E. T., Sánchez, J., de C Filho, J. A., and Viana, W. (2016). Design and 
V Congresso Brasileiro de Informática na Educação (CBIE 2016)

Anais do XXVII Simpósio Brasileiro de Informática na Educação (SBIE 2016)

usability of a braille-based mobile audiogame environment. In Proceedings of the 31st Annual ACM Symposium on Applied Computing, pages 232-238. ACM.

[Cheiran and Pimenta 2011] Cheiran, J. F. P. and Pimenta, M. S. (2011). "eu tambem quero jogar!": Reavaliando as praticas e diretrizes de acessibilidade em jogos. In Proceedings of the 10th Brazilian Symposium on on Human Factors in Computing Systems and the 5th Latin American Conference on Human-Computer Interaction, IHC+CLIHC '11, pages 289-297, Porto Alegre, Brazil, Brazil. Brazilian Computer Society.

[de Souza et al. 2012] de Souza, M. d. F. C., de Castro Filho, J. A., and Andrade, R. M. (2012). Customização guiada: uma estratégia orientada a modelos para a produção de objetos de aprendizagem. In Anais do Simpósio Brasileiro de Informática na Educação, volume 23.

[DIS 2009] DIS, I. (2009). 9241-210: 2010. ergonomics of human system interaction-part 210: Human-centred design for interactive systems. International Standardization Organization (ISO). Switzerland.

[IBGE 2010] IBGE (2010). Censo demográfico - pessoas com deficiência. http://www.ibge.gov.br/estadosat/temas.php?sigla=rj\&tema= censodemog2010_defic. Acessado em 24-maio-2016.

[Maria C. C. Araújo 2015] Maria C. C. Araújo, A. Façanha, T. G. R. D. S. J. R. M. C. A. V. W. (2015). Um estudo das recomendações de acessibilidade para audiogames móveis. In XIV Simpósio Brasileiro de Jogos e Entretenimento Digital - ISSN: 2179 2259, pages 610-617. SBC.

[Meira et al. 2008] Meira, J. N. B., Ferracini, C. C., Gimenes, A. L. M., Neves, F. H. D., Simonassi, R., and Pimentel, E. P. (2008). Uma ferramenta de autoria de materiais instrucionais com símbolos matemáticos acessíveis a deficientes visuais. Anais do XIX Simpósio Brasieliro de Informática na Educação.

[Milne et al. 2014] Milne, L. R., Bennett, C. L., Ladner, R. E., and Azenkot, S. (2014). BraillePlay: Educational smartphone games for blind children. In Proceedings of the 16th International ACM SIGACCESS Conference on Computers \& Accessibility, ASSETS '14, pages 137-144, New York, NY, USA. ACM.

[Silva et al. 2014] Silva, A. R. S., Façanha, A. R., Viana, W., de Castro Filho, J. A., and Sánchez, J. (2014). Especificação e desenvolvimento de um ambiente educativo móvel para a prática da escrita braille. In Anais do Simpósio Brasileiro de Informática na Educação, volume 25, page 431.

[Tarouco et al. 2004] Tarouco, L. M., Fabre, M., Grando, A. R., and Konrath, M. L. (2004). Objetos de aprendizagem para m-learning. In Florianópolis: SUCESU-Congresso Nacional de Tecnologia da Informação e Comunicação.

[Thorpe et al. 2011] Thorpe, A., Ma, M., and Oikonomou, A. (2011). History and alternative game input methods. In Computer Games (CGAMES), 2011 16th International Conference on, pages 76-93. IEEE.

[U. RITTERFELD and Wong. 2009] U. RITTERFELD, C. SHEN, H. W. L. N. and Wong., W. (2009). Multimodality and interactivity: Connecting properties of serious games with educational outcomes. Cyberpsychology \& Behavior, 12(6):691-697. 